\title{
The antitumor effects of oncolytic adenovirus H101 against lung cancer
}

\author{
JIE LEI ${ }^{1}$, QI-HUA LI ${ }^{2}$, JU-LUN YANG ${ }^{1}$, FENG LIU $^{3}$, LI WANG $^{1}$, WEN-MANG XU ${ }^{1}$ and WEN-XING ZHAO ${ }^{1}$ \\ ${ }^{1}$ Department of Pathology, Kunming General Hospital of PLA, Kunming, Yunnan 650032; \\ ${ }^{2}$ College of Science and Technology, Yunnan Agriculture University, Kunming, Yunnan 650201; \\ ${ }^{3}$ School of Life Sciences of Yunnan University, Kunming, Yunnan 650091, P.R. China
}

Received March 19, 2015; Accepted May 4, 2015

DOI: 10.3892/ijo.2015.3045

\begin{abstract}
Lung cancer is the leading cause of cancer mortality in both men and women, with dismal survival rates due to latestage diagnoses and a lack of efficacious therapies. The new treatment options with completely novel mechanism of therapeutic activity are needed for lung cancer to improve patient outcome. The present study was aimed at testing the efficacy of recombinant adenovirus $\mathrm{H} 101$ as an oncolytic agent for killing human lung cancer cell lines in vitro and in vivo. We assessed the coxsackievirus adenovirus receptor (CAR) expression on human lung cancer cell lines by RT-PCR and immunocytochemistry staining. Viral infectivity and viral replication in lung cancer cells was assayed by flow cytometry and real-time fluorescent quantitative PCR. After H101 treatment, cytotoxic effect, cell cycle progression and apoptosis were further examined by lactate dehydrogenase release assay and flow cytometry in vitro, respectively. In vivo, antitumor effects of H101 were assessed on SCID Beige mice xenografted with human lung cancer cells. Receptor characterization confirmed that human lung cancer cell lines expressed CAR receptor for adenovirus type 5 . Lung cancer cells were sensitive to infection by the H101 virus. H101 infection and replication resulted in very potent cytotoxicity, G2/M phase arrest and cell lysis. In vivo, we also showed that $\mathrm{H} 101$ significantly inhibited tumor growth following intratumoral injection, with virus replication, cell degeneration and necrosis in the tumor tissue. These results have important implications for the treatment of human lung cancer.
\end{abstract}

\section{Introduction}

Worldwide, lung cancer is the leading cause of cancer mortality. Non-small cell lung cancer (NSCLC) accounts for

Correspondence to: Dr Wen-Xing Zhao, Department of Pathology, Kunming General Hospital of PLA, 212 Daguan Road, Kunming, Yunnan 650032, P.R. China

E-mail: wenxingzhao@sohu.com

Key words: lung cancer, oncolytic virus, H101, coxsackievirus adenovirus receptor, gene therapy $\sim 85 \%$ of all lung cancers. Standard first-line treatment options for NSCLC depend on disease and patient characteristics and may include surgery, platinum-based doublet chemotherapy and radiotherapy (1). Although surgical resection is curative if diagnosis occurs at early stage I or stage II disease, $70 \%$ of all newly diagnosed patients present with local advanced or metastatic disease and require systemic chemotherapy $(1,2)$. Chemotherapy combinations for more advanced diseases have shown to convey no benefit on overall survival or quality of life beyond 4-6 cycles $(3,4)$. NSCLC patients with epidermal growth factor receptor (EGFR) mutations initially respond to EGFR tyrosine kinase inhibitors, however, most patients experience a relapse within 1 year $(5,6)$. Despite the development of novel molecular therapies, the prognosis of lung cancer is still poor and the 5-year survival remains $<20 \%$ (7-9). Hence, novel and more effective approaches are needed for the treatment of advanced lung cancer.

Oncolytic viruses represent an emerging therapeutic platform for the treatment of human cancer with unique attributes compared with conventional therapeutic modalities, which is able to selectively infect and lyse tumor cells where after the released progeny virions reinfect neighboring tumor cells and also enter the blood stream to infect metastasized tumor cells, while ideally leaving normal cells unharmed (10). Among them, adenoviruses were widely used as oncolytic viral agents in cancer therapy, as they possess an inherent potential to kill the cells that sustain their replication, and they are considered to be safe and have been used in several clinical settings (11-13). To restrict cytocidal effect to tumor cells, the conditionally replicative adenoviruses (CRAds) have been developed to restrict viral replication to target cancerous tissues and inhibit replication in normal healthy cells. This has been attempted by exploiting loss-of-function mutations in viral sequences, or linking genes E1A/E1B to cancer-specific promoters, such as the telomerase or prostate-specific rat probasin promoters or the human prostate-specific enhancer/promoter $(14,15)$.

$\mathrm{H} 101$, a conditionally replicative adenovirus, was generated by both E1B and $E 3$ gene deletion, which selectively infects and kills tumor cells through viral oncolysis (16). Without E1B to inactivate p53, H101 adenovirus cannot replicate and lyse normal cells where p53 is active, which does not have significant cytopathic effects on normal cells (17). In addition, the deletion of a 78.3-85.8-nm gene segment in the E3 region, 
which encodes the adenovirus death protein, may improve the safety of the product (5). In China, H101 has been clinically approved for the treatment of several malignancies (5).

In the present study, we describe the CAR expression of lung cancer cells and cytopathologic effects of H101 infection on cancer cells in vitro, and we further demonstrate that H101 virus suppressed lung cancer xenografts growth in vivo.

\section{Materials and methods}

Cell culture and recombinant adenovirus H101. XWLC-05, human lung adenocarcinoma cell line, was kindly provided as a gift by Kunming College University, which was established from the primary tumor of a Xuanwei woman with lung adenocarcinoma (18). The human embryonic kidney cell line HEK293A and lung squamous cell carcinoma SK-MES-1 were purchased from the Cell Bank of Kunming Institute of Zoology, Chinese Academy of Sciences. All cell lines were cultured in Dulbecco's modified Eagle's medium (DMEM) supplemented with $10 \%$ fetal bovine serum (FBS), $100 \mathrm{U} / \mathrm{ml}^{-1}$ penicillin and $100 \mu \mathrm{g} / \mathrm{ml}^{-1}$ streptomycin in a humidified atmosphere with $5 \% \mathrm{CO}_{2}$ at $37^{\circ} \mathrm{C}$. Recombinant adenovirus H101 was obtained from Shanghai Sunway Biotech Co., Ltd. (Shanghai, China).

Measurement of coxsackievirus adenovirus receptor (CAR) expression. CAR expression of lung cancer was analyzed as previously described (19). In brief, cells growing on cover glass were incubated with the mouse monoclonal anti-CAR antibody (1:50; Santa Cruz Biotechnology, Santa Cruz, CA, USA) in binding buffer at $4^{\circ} \mathrm{C}$ overnight. Afterwards, the immune reaction was visualized using the EnVision ${ }^{\mathrm{TM}}$ detection system (Dako, Glostrup, Denmark). Analysis of stained cells was performed under a microscope.

For RT-PCR detection of CAR mRNA expression, total RNA was extracted from cells by using RNAiso Plus kit (Takara, Dalian, China). The cDNA was synthesized by using First Strand cDNA Synthesis kit (Invitrogen, Carlsbad, CA, USA). Oligonucleotide primers with the following sequences were used: CAR, forward primer, TTCAGGTGCGAGATG TTA and reverse primer, GAATGATTACTGCCGATG; GAPDH, forward primer, AGAAGGCTGGGGCTCATTTG and reverse primer, AGGGGCCATCCACAGTCTTC. The PCR was performed by using the following parameters: $94^{\circ} \mathrm{C}$ $5 \mathrm{~min} ; 94^{\circ} \mathrm{C} 30 \mathrm{sec}, 56^{\circ} \mathrm{C} 30 \mathrm{sec}, 72^{\circ} \mathrm{C} 30 \mathrm{sec}$ x 35 cycles; $72^{\circ} \mathrm{C}$ $7 \mathrm{~min}$. PCR product was visualized by $1 \%$ agarose gel electrophoresis using $0.5 \mu \mathrm{g} / \mathrm{ml}$ ethidium bromide.

Quantification of infection efficiency. To assess the susceptibility to H101 infection of the XWLC-05 cell lines, cells were plated at a density of $10^{6}$ cells/well in 6 -well plates. Six hours after seeding, the cells were infected with adenoviruses harboring the green fluorescent protein reporter gene (Ad-GFP) at multiplicity of infection (MOI) of 1, 10, 100 and 1000 for $2 \mathrm{~h}$ at $37^{\circ} \mathrm{C}$. At the end of the incubation period, the virus was removed and the cells were maintained in their standard medium. Infected cells expressing GFP were identified $48 \mathrm{~h}$ after infection using a fluorescent microscope (Nikon, Tokyo, Japan) and BD Accuri C6 flow cytometry (BD Biosciences, San Jose, CA, USA). The mean ratio of infected cancer cells was calculated. Results are presented as the means \pm SD of at least three independent experiments.

Real-time fluorecent quantitative PCR for viral Hexon mRNA. XWLC-05 was infected with oncolytic virus H101 at a MOI of 100 as described above. At 24, 48 and 72 h post-infection, virus replication was evaluated by studying relative changes of viral Hexon mRNA expression. Total RNA was extracted from cells by using RNAiso Plus kit (Takara). cDNA was synthesized by using First Strand cDNA Synthesis kit (Invitrogen) according to the manufacturer's instructions. Gene expression was quantified by real-time quantitative PCR using SYBR ${ }^{\circledR}$ Premix Ex Taq $^{\mathrm{TM}}$ II (Takara) and primers recognizing Hexon as previously described (20).

Virus-mediated cytotoxicity assays. Cells were plated in 96-well plates at $2 \times 10^{4}$ cells/well. After $6 \mathrm{~h}$, cells were infected with H101 at MOI of 1, 10, 100 and 1000 or Ad-GFP at a MOI of 100 , or vehicle treatment (phosphate-buffer saline, PBS). Virus-induced cytotoxicity was analyzed daily for 5 days by measuring the release of lactate dehydrogenase (LDH) in conditioned media using the CytoTox $96^{\circledR}$ Non-Radioactive Cytotoxicity assay kit (Promega, Madison, WI, USA) and by spectrophotometry (Bio-Rad Laboratories, Hercules, CA, USA) at $490 \mathrm{~nm}$. Percent cytotoxicity $=1-100 \% \times$ [(experimental LDH release - spontaneous LDH release)/(maximum LDH release - spontaneous LDH release)]. Samples were measured in triplicate.

Cell cycle and cell apoptosis detection. For cell cycle detection, cells were seeded at $10^{5}$ cells/well in 6-well plates. Cells were harvested at 24,48 and 72 h post-infection with $\mathrm{H} 101$ $(\mathrm{MOI}=100)$ or Ad-GFP $(\mathrm{MOI}=100)$ or vehicle treatment (PBS). Cells were washed twice with PBS and stained with $10 \mu \mathrm{g} / \mathrm{ml}$ propidium iodile (PI). The samples were analyzed with BD Accuri C6 flow cytometry to determine cell cycle distribution. Apoptosis was quantified by detecting surface exposure of phosphatidylserine in apoptotic cells using the Annexin V-FITC/PI apoptosis detection kit (BD Biosciences Clontech, CA, USA). Cancer cells were infected for $24 \mathrm{~h}$ with H101 (MOI=100), apoptotic cells were detected according to the manufacturer's instruction, using BD Accuri C6 flow cytometry.

Mouse procedures. Animal experiments were approved by and performed in accordance with institutional guidelines of the Yunnan Animal Care and Use Committee. Immunodeficient homozygous SCID Beige male mice, 7-8 weeks of age, were obtained from Vital River Laboratories (Beijing, China). Back subcutaneous tumors were established by injection of $5 \times 10^{6}$ XWLC-05 cells in $100 \mu$ of PBS. When tumor volume averaged $\sim 100 \mathrm{~mm}^{3}, 1 \times 10^{8}$ PFU of H101 in $100 \mu \mathrm{l}$ was injected in tumor daily for 4 days. Tumor dimensions were measured by caliper every 4 days for 28 days, and tumor volume (V) was estimated by the formula (long diameter $\mathrm{x}$ short diameter $\left.{ }^{2}\right) / 2$. The tumor growth inhibition rate was calculated according to the previously described method (21). Mice were sacrificed at 32 days from the first H101 injection, tumors and other important organism were harvested, fixed in formalin and embedded in paraffin. Sections were deparaffinized with xylene, hydrated 

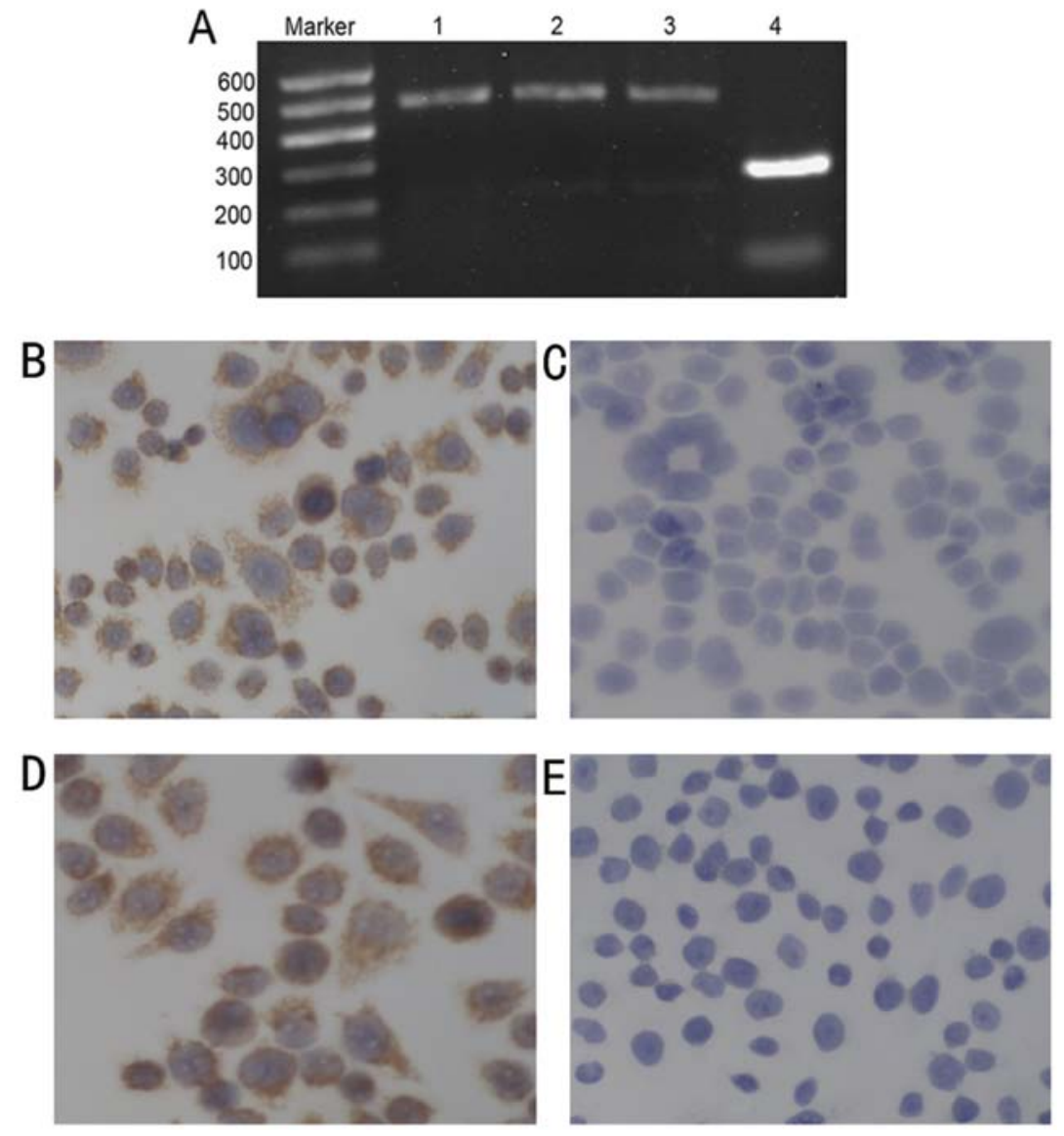

Figure 1. Lung cancer cells express coxsackievirus adenovirus receptor. (A) RT-PCR results showed CAR mRNA specific transcription in XWLC-05, SK-MES-1 and HEK-293A cell lines. Lane 1, XWLC-05; lane 2, SK-MES-1; lane 3, HEK-293A, positive control; lane 4, GAPDH. Typical results for CAR immunocytochemistry staining are shown in XWLC-05 cell line (B) and control (C), SK-MES-1 cell line (D) and control (E) (magnification, x200). Abundant CAR presence in lung cancer cells was revealed.

in ethanol and distilled water, and stained with hematoxylin and eosin $(\mathrm{H} \& \mathrm{E})$. Tumor sections were also evaluated for Hexon protein expression of H101 viruses using FITC conjugated mouse anti-Hexon monoclonal antibody (GeneTex, Inc., Irvine, CA, USA), nuclei were counterstained with DAPI and the sections visualized by fluorescent microscope (Olympus, Tokyo, Japan).

\section{Results}

Lung cancer cells express CAR-specific mRNA and protein. Ad enters a cell via receptor-mediated endocytosis involving the binding of the fiber knob of viral capsid proteins to the primary receptor CAR. We investigated the CAR mRNA expression on lung cancer cell lines by RT-PCR assays. The results showed that both XWLC-05 and SK-MES-1 expressed CAR mRNA similarly to 293 cells (Fig. 1A). Using immunocytochemistry, we also analyzed the presence of CAR protein in XWLC-05 (Fig. 1B) and SK-MES-1 cells (Fig. 1D). RT-PCR and immunochemistry analysis confirmed that lung cancer cells expressed CAR, which provided a molecular base for oncolytic adenovirus H101 infection.

The susceptibility of XWLC-05 cells to H101 infection. As the adenovirus type 5 infected cells use common mechanism, the susceptibility of lung cancer cells to infection with Ad5 was determined by using Ad-GFP. As illustrated in Fig. 2, infected cancer cells expressing GFP were visualized using a fluorescent microscope (Fig. 2A), and flow cytometric analysis revealed that the percentage of Ad-GFP positive cells was increased at a dose-dependent manner (Fig. 2B and C). These results indicated that Ad-GFP transduced XWLC-05 efficiently.

Replication of oncolytic adenovirus $\mathrm{H} 101$ in lung cancer cells. Since adenovirus transduced XWLC-05 cells efficiently, we investigated the replicative potential of H101 in the cancer cells. XWLC-05 cells were infected with H101 virus and analyzed for the amount of Hexon mRNA by real-time fluorescent quantitative PCR. Results showed that H101 replicated efficiently in XWLC-05 cells with comparative Hexon mRNA copy number increased in the cells time-dependently (Fig. 3). Compared with 24 h, Hexon mRNA was significantly increased by 5.2 -fold at $48 \mathrm{~h}$ and 27.4 -fold at $72 \mathrm{~h}$. Increases in Hexon mRNA between 24 and $72 \mathrm{~h}$ post-infection were indicative of viral replication. These results indicated that the H101 virus was able to replicate efficiently in lung cancer cells.

Cytopathic effects of H101 on XWLC-05 cells. XWLC-05 cells were used to further investigate the cytopathic changes triggered by H101 infection. The cytopathic effect was 

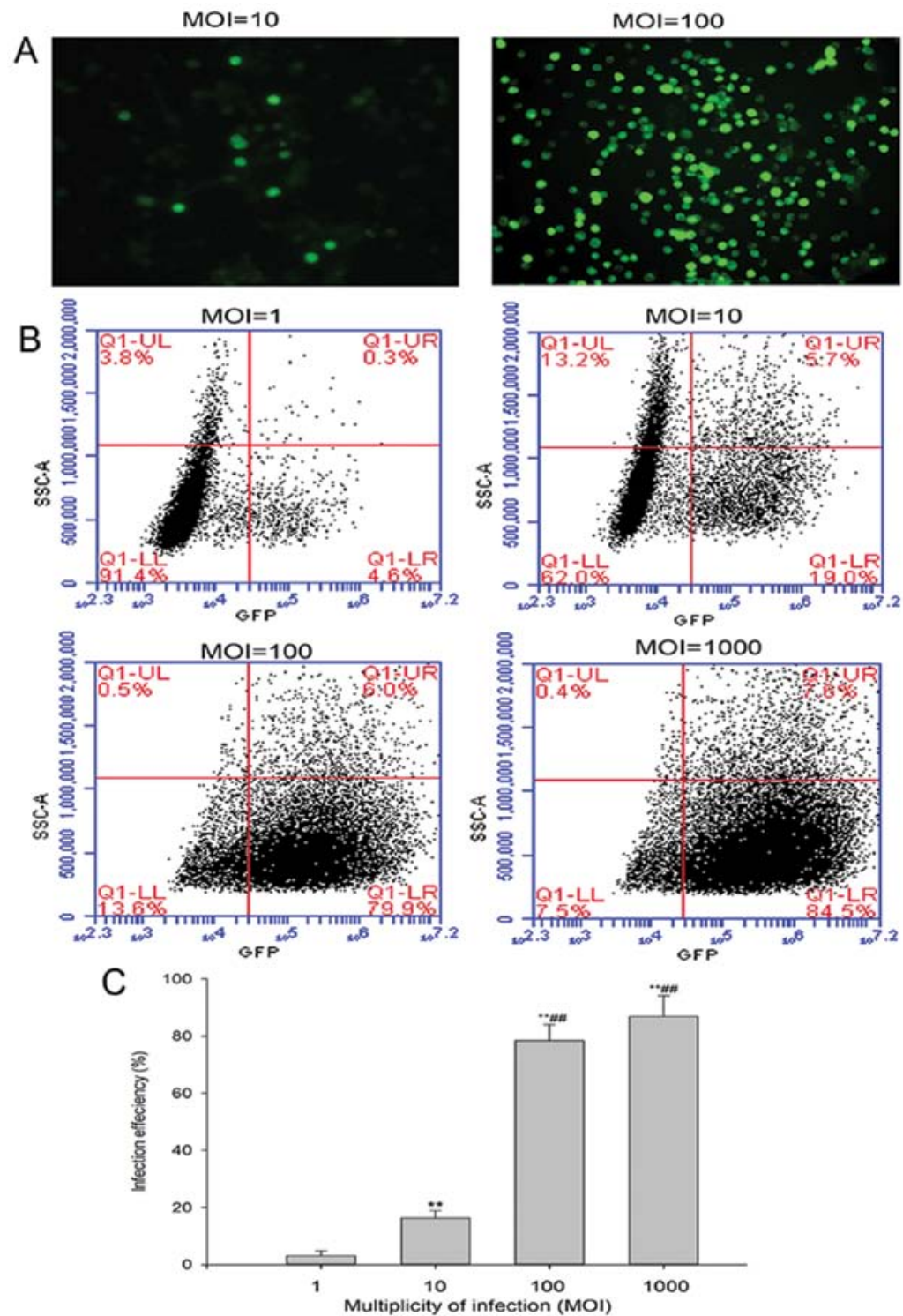

Figure 2. Adenovirus infection of XWLC-05. XWLC-05 cells were infected with Ad-GFP at indicated MOIs. At $48 \mathrm{~h}$ post-infection, infected cells expressing GFP were visualized using a fluorescent microscope (A) and analyzed by flow cytometry (B). (C) Results are shown as the positive rate of infected tumor cells. XWLC-05 cells showed susceptibility to adenovirus infection in vitro. The mean ratio of infected cancer cells is presented as the means \pm SD of at least three independent experiments. ${ }^{* *} \mathrm{P}<0.01$ significantly different vs. $\mathrm{MOI}=1,{ }^{\# \prime} \mathrm{P}<0.01$ significantly different vs. MOI=10.

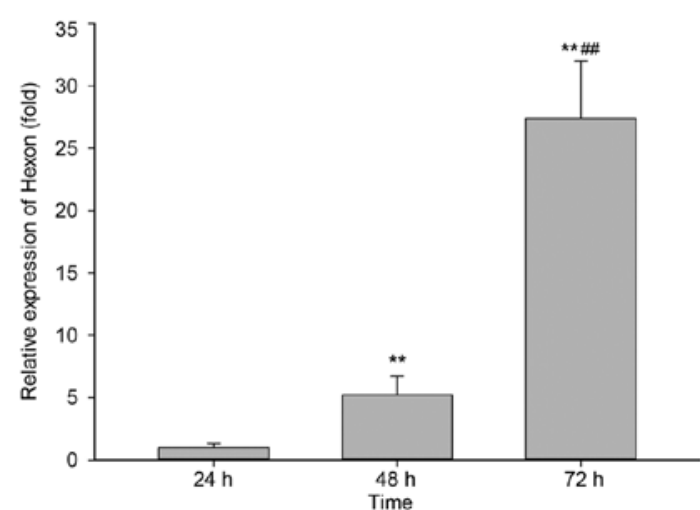

Figure 3. The replication of H101 virus in XWLC-05 cells. XWLC-05 cells were infected with $\mathrm{H} 101$ at MOI of 100, the Hexon mRNA was determined by real-time quantitative PCR. The replication of H101 in XWLC-05 was demonstrated by Hexon mRNA transcription increase at a time-dependent manner. Results are presented as the means $\pm \mathrm{SD}$ of at least three independent experiments. ${ }^{* *} \mathrm{P}<0.01$ significantly different vs. $24 \mathrm{~h},{ }^{\# \#} \mathrm{P}<0.01$ significantly different vs. $48 \mathrm{~h}$.

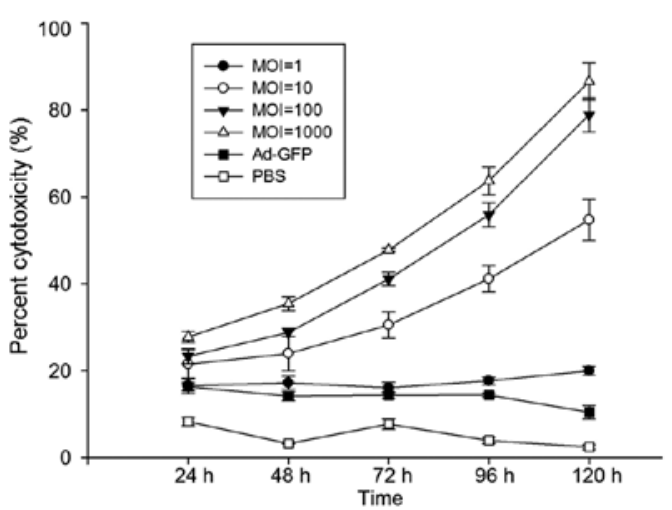

Figure 4. Cytotoxic effect of H101 virus on XWLC-05 cells. XWLC-05 cells were treated with $\mathrm{H} 101$ at the indicated MOI or Ad-GFP at a MOI of 100 or vehicle (PBS). Virus-induced cytotoxicity was analyzed by measuring the release of lactate dehydrogenase (LDH) in the conditioned media. The H101-mediated cytotoxicity was increased in a time- and dose-dependent manner. Results are presented as the means \pm SD of at least three independent experiments. 


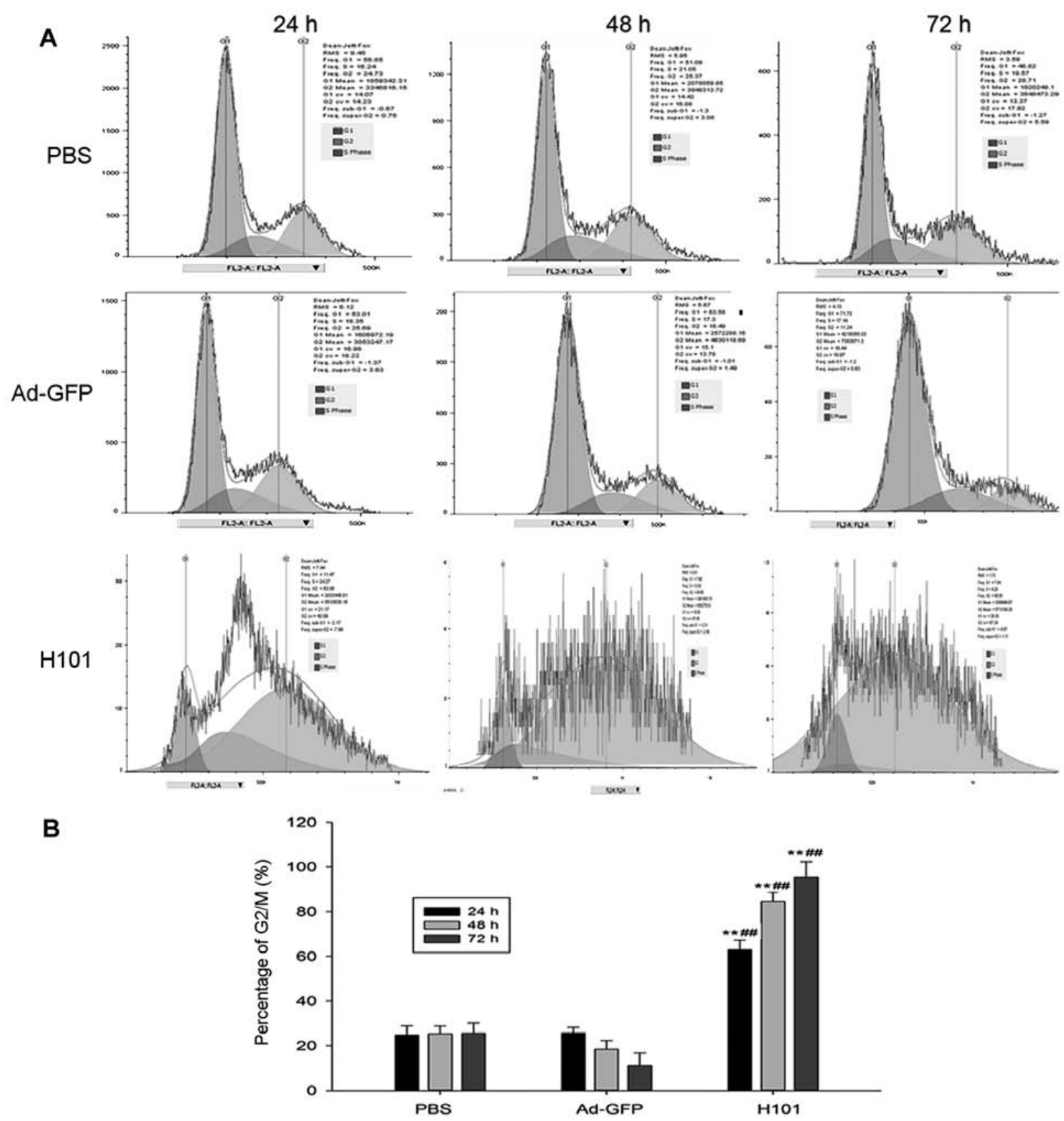

Figure 5. Effects of H101 infection on cell cycle progression in XWLC-05 cells. Cells were treated with H101 or Ad-GFP or vehicle (PBS), the distribution of cells in different phases of cell cycle was analyzed by flow cytometry after propidium iodide staining (A). The percentage of cells in G2/M cell cycle phase is shown (B). It indicates H101 infection induced G2/M arrest with a marked increase of percentage of $\mathrm{G} 2 / \mathrm{M}$. ${ }^{* *} \mathrm{P}<0.01$ significantly different vs. PBS, ${ }^{* \#} \mathrm{P}<0.01$ significantly different vs. Ad-GFP.

evaluated by viral cytotoxicity, cell cycle progression and apoptosis analysis. In agreement with the above results, $\mathrm{LDH}$ assays indicated that H101 killed XWLC-05 cells efficiently with LDH increases in a time- and dose-dependent manner (Fig. 4). Microscopy examination also revealed nuclear swelling and DNA release from the nucleus into the cytosol at later time-points after infection, indicating that most cells died of necrosis (data not shown). In order to understand the underlying mechanisms of cytopathic effect by H101 treatment, we analyzed its effects on cell cycle phase distribution and apoptosis in XWLC-05 cells. As illustrated in Fig. 5, H101 induced strong accumulation of cells in the G2/M phase in XWLC-05 cells at a MOI of 100 after 24-, 48- or 72-h treatments, which was associated with parallel depletion of cells in the G0/G1 phase (Fig. 5A). Compared to vehicle or Ad-GFP, the percentage of G2/M phase cells in H101 infected cells was significantly different at all time-points (Fig. 5B). However, the percentage of apoptotic cells was very low and not significantly different at $24 \mathrm{~h}$ post-infection by propidium iodide/Annexin $\mathrm{V}$ staining analysis (data not shown). The results suggest that $\mathrm{H} 101$ infection induced cytotoxic effects on XWLC-05 cells by cell cycle arrest and cell necrosis.

H101 inhibited the growth of subcutaneous XWLC-05 xenografts. In vitro, we investigated the oncolytic effect of H101 on XWLC-05 cells, which significantly induced cytotoxicity in 

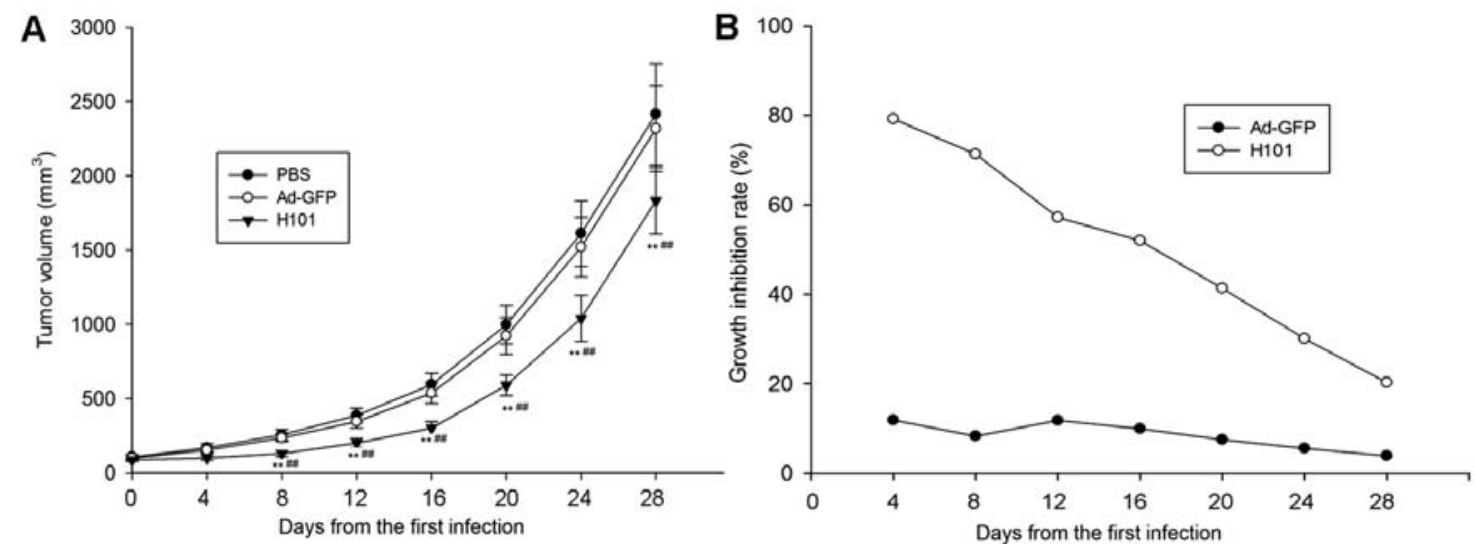

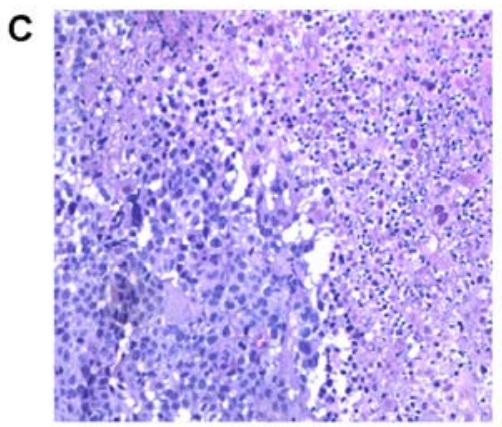

$\mathrm{H} 101$

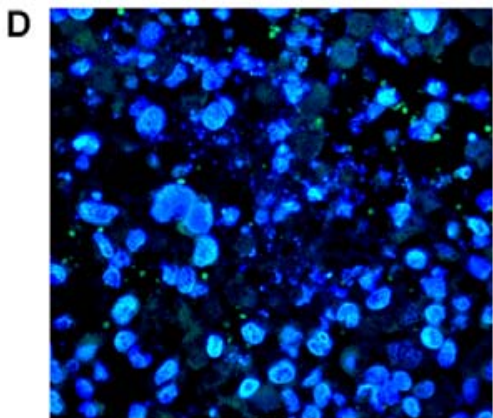

$\mathrm{H} 101$

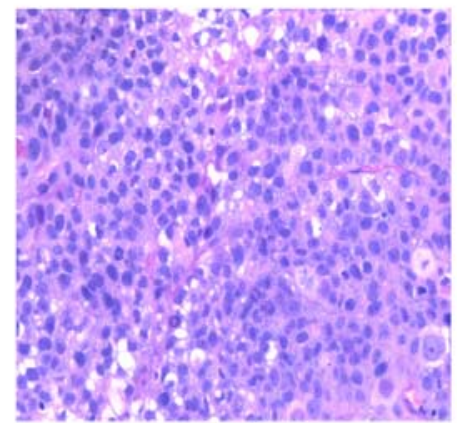

Ad-GFP

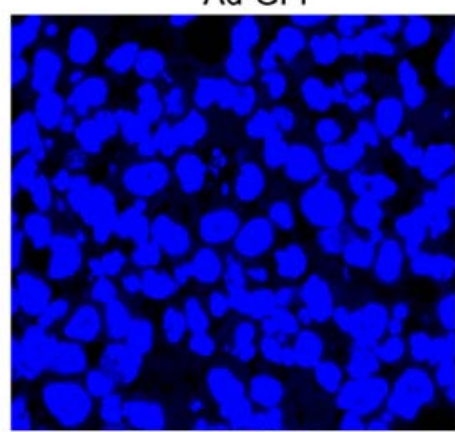

Ad-GFP

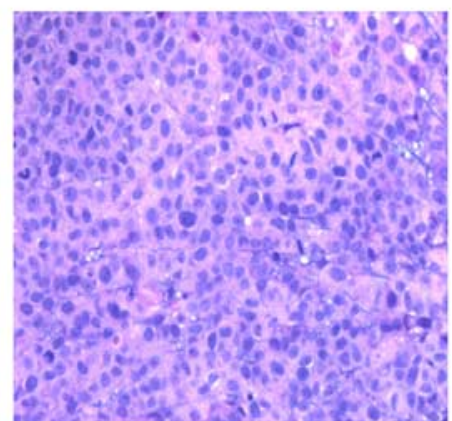

PBS

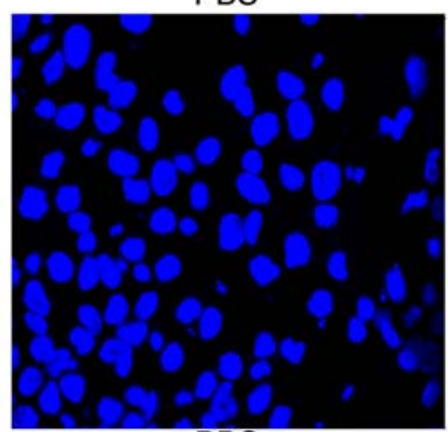

PBS

Figure 6. H101 suppresses the growth of XWLC-05 lung tumors in mice. (A) Subcutaneous XWLC-05 xenografts were established in SCID Beige mice and treated with intratumoral injections of H101, Ad-GFP or PBS. Results showed that H101 virus significantly suppressed growth of XWLC-05 xenografts. ${ }^{* *} \mathrm{P}<0.01$ significantly different vs. $\mathrm{PBS},{ }^{\# \#} \mathrm{P}<0.01$ significantly different vs. Ad-GFP. (B) Growth inhibition rate of tumor in $\mathrm{H} 101$ group was markedly increased as compared with Ad-GFP group and it gradually decreased. (C) Typical histological appearance showed H101 induced necrosis of lung cancer cells with condensed nuclei (magnification, x200). (D) Immunofluorescence assay indicated marked Hexon protein expression in tumors treated with H101 and no Hexon protein expression in tumors treated with Ad-GFP or PBS.

XWLC-05 cells as compared to Ad-GFP or vehicle control. We next evaluated the susceptibility of these cells to H101 treatment in vivo. H101 significantly suppressed the growth of XWLC-05 tumor xenografts as compared to Ad-GFP or PBS from 8 day to 28 day end-point of the study (Fig. 6A). The growth inhibition rate of the H101-treated group was markedly increased as compared with Ad-GFP group, and it gradually decreased due to limited viral replication and propagation of the entire tumor mass (Fig. 6B). Histological examination of Ad-GFP or PBS-treated tumors revealed healthy cancer cells arranged in tumor architecture. However, in H101 virustreated group, H101 induced cytocidal effects on XWLC-05 cells, which were characterized by scattered necrotic patches surrounded by healthy tumor cells, indicative of incomplete oncolysis due to limited viral replication and propagation of cancer cells (Fig. 6C). To associate cell death and active viral infection, immunofluorescence (IF) staining with FITC anti-Hexon was performed on paraffin-embedded sections collected 28 days after virus injection, and IF staining showed the presence of viral proteins in the tumor cells of H101 virustreated group (Fig. 6D). In general, H101 inhibited the growth of subcutaneous XWLC-05 xenografts by virus replication and virus-induced cytotoxicity in vivo.

\section{Discussion}

Contrary to the improved survival outcomes for many other types of cancers, the prognosis for people diagnosed with lung cancer remains poor despite conventional therapy with surgery, chemotherapy and new molecular target therapies, with 5-year relative survival being $\sim 6-14 \%$ among males and $7-18 \%$ among females (2). This poor survival means that new 
treatment options with completely novel mechanisms of therapeutic activity are needed for lung cancer patients to improve outcome.

Oncolytic virotherapy have proposed as a promising therapeutic approach to fight cancer. A variety of viruses have shown oncolytic properties including adenovirus, herpes simplex virus, Newcastle disease virus, vesicular stomatitis virus and reovirus (22). Among a variety of oncolytic viral agents, CRAds specifically aimed at killing tumor cells while sparing normal cells have been developed as new agents for cancer therapy $(23,24)$, and demonstrated efficacy and safety in preclinical $(25,26)$ and clinical trials $(27-29)$. Various methods have been used to achieve a selective expression. These approaches included the use of tumor-specific promoters to drive E1A gene expression (30) or the E1B deletion, which restricts the oncolytic activity to p53-defective tumor cells. ONYX-015 was the first to be tested in clinical trials, revealing itself as a well-tolerated and safe tool and a promising therapeutic agent in cancer (31).

In the present study, we describe a novel application of adenovirus H101 in lung cancer, which was very similar to ONYX-015. H101 was generated by deleting both E1B and E3 genes of adenovirus type 5 (Ad5), which selectively infects and kills tumor cells through viral oncolysis while leaving normal cells unharmed without E1B to inactivate P53, and improves the safety of the product with the E3 deletion. Previous reports have shown the therapeutic potentials in other tumors. In clinic, a multicenter randomized phase 3 clinical trial showed that the combination therapy with H101 for head and neck squamous cell carcinoma and esophageal cancer yielded a $27 \%$ increase in overall response rates compared with fluorouracil plus cisplatin-based chemotherapy alone (22). This virus was approved by the Chinese State Food and Drug Administration for the treatment of late-stage refractory nasopharyngeal cancer.

We investigated the CAR mRNA and protein expression in a lung cancer cell line, as CAR is the entry gateway for adenovirus and needed for efficient adenovirus-mediated oncolysis. Our finding clearly indicated the high CAR mRNA and protein expression in lung cancer cells including adenocarcinoma XWLC-05 and squamous cell carcinoma SK-MES-1. Therefore, it is speculated that lung cancer may have susceptibility to adenovirus $\mathrm{H} 101$ infection. In a previous study, high CAR mRNA and protein expression was observed in other tumors such as breast cancer and early-stage oesophageal adenocarcinoma (19,32). Functionally, CAR may promote early carcinogenesis as suggested for cervix and ovarian cancers, with CAR-expressing cell lines displaying less sensitivity towards apoptotic stimuli (33). As for lung cancer cells, it was suggested that CAR might be required for effective xenograft formation $(34,35)$.

We next demonstrated that adenovirus H101 was able to efficiently infect and replicate in XWLC-05 cell lines in vitro, leading to cell growth stress and oncolysis. H101 infection induced the accumulation of $\mathrm{G} 2 / \mathrm{M}$ phase cells, and nuclear swelling and DNA release from the nucleus into cytosol at late time-points, which indicated that the underlying mechanism for cytopathic effects of H101 involved cell cycle stress and necrosis, which is in agreement with a previous study (20).

In vivo, we showed that the tumor volume of mice bearing XWLC-05 xenograft was significantly reduced compared with mock controls when treated with H101. For safe and effective virotherapy, target tissue-restricted virus expression is desirable. In the present study, the intratumoral injection of $\mathrm{H} 101$ in XWLC-05 xenografts exhibited localized intratumoral Hexon protein expression. Moreover, there was no evidence of viral spread to any other organs including brain, liver, heart and gut, based on Hexon protein expression under a fluorescence microscope (data not shown), indicating tumor-specific viral infection and activity. Although oncolytic adenovirus H101 are highly efficient at killing tumor cells in vitro, the activity in vivo was moderate, and the tumor growth inhibition gradually decreased due to limited viral proliferation and rapid propagation of cancer cells. In other studies, the results exhibited relative activities of oncolytic virus in animals and the clinic, thus, combination therapy is often suggested for therapeautic potential in vivo. For example, others have reported previously that radiation can induce greater vector replication, causing increased oncolytic activity $(36,37)$. Based on our results, it would be of significant clinical importance to evaluate the effect of combination therapy of H101 and chemotherapy or radiation.

In conclusion, the present study demonstrates therapeutic potential of adenovirus H101 in lung cancer, which provides novel insights into the treatment of lung cancer using the oncolytic adenovirus.

\section{Acknowledgements}

The present study was supported by the Fundamental Research Funds (2010ZC186) and the Reserve Talent Funds (2008Y003) of Yunnan Province.

\section{References}

1. Molina JR, Yang P, Cassivi SD, Schild SE and Adjei AA: Non-small cell lung cancer: Epidemiology, risk factors, treatment, and survivorship. Mayo Clin Proc 83: 584-594, 2008.

2. Ramalingam SS, Owonikoko TK and Khuri FR: Lung cancer: New biological insights and recent therapeutic advances. CA Cancer J Clin 61: 91-112, 2011.

3. Roy M, Luo YH, Ye M and Liu J: Nonsmall cell lung cancer therapy: Insight into multitargeted small-molecule growth factor receptor inhibitors. BioMed Res Int 2013: 964743, 2013.

4. Lorigan P, Verweij J, Papai Z, Rodenhuis S, Le Cesne A, Leahy MG, Radford JA, Van Glabbeke MM, Kirkpatrick A, Hogendoorn PC, et al; European Organisation for Research and Treatment of Cancer Soft Tissue and Bone Sarcoma Group Study: Phase III trial of two investigational schedules of ifosfamide compared with standard-dose doxorubicin in advanced or metastatic soft tissue sarcoma: A European Organisation for Research and Treatment of Cancer Soft Tissue and Bone Sarcoma Group Study. J Clin Oncol 25: 3144-3150, 2007.

5. Herbst RS, Heymach JV and Lippman SM: Lung cancer. N Engl J Med 359: 1367-1380, 2008

6. Carrera S, Buque A, Azkona E, Aresti U, Calvo B, Sancho A, Arruti M, Nuño M, Rubio I, de Lobera AR, et al: Epidermal growth factor receptor tyrosine-kinase inhibitor treatment resistance in non-small cell lung cancer: Biological basis and therapeutic strategies. Clin Transl Oncol 16: 339-350, 2014.

7. Jemal A, Center MM, DeSantis C and Ward EM: Global patterns of cancer incidence and mortality rates and trends. Cancer Epidemiol Biomarkers Prev 19: 1893-1907, 2010.

8. Jemal A, Siegel R, Ward E, Hao Y, Xu J, Murray T and Thun MJ: Cancer statistics, 2008. CA Cancer J Clin 58: 71-96, 2008.

9. Pao W and Girard N: New driver mutations in non-small-cell lung cancer. Lancet Oncol 12: 175-180, 2011. 
10. Guo ZS, Thorne SH and Bartlett DL: Oncolytic virotherapy: Molecular targets in tumor-selective replication and carrier cellmediated delivery of oncolytic viruses. Biochim Biophys Acta 1785: 217-231, 2008.

11. Jiang G, Yang CS, Xu D, Sun C, Zheng JN, Lei TC and Liu YQ: Potent anti-tumour activity of a novel conditionally replicating adenovirus for melanoma via inhibition of migration and invasion. Br J Cancer 110: 2496-2505, 2014.

12. Immonen A, Vapalahti M, Tyynelä K, Hurskainen H, Sandmair A, Vanninen R, Langford G, Murray N and Ylä-Herttuala S: AdvHSV-tk gene therapy with intravenous ganciclovir improves survival in human malignant glioma: A randomised, controlled study. Mol Ther 10: 967-972, 2004.

13. Khuri FR, Nemunaitis J, Ganly I, Arseneau J, Tannock IF, Romel L, Gore M, Ironside J, MacDougall RH, Heise C, et al: A controlled trial of intratumoral ONYX-015, a selectivelyreplicating adenovirus, in combination with cisplatin and 5 -fluorouracil in patients with recurrent head and neck cancer. Nat Med 6: 879-885, 2000.

14. Doloff JC and Waxman DJ: Adenoviral vectors for prodrug activation-based gene therapy for cancer. Anticancer Agents Med Chem 14: 115-126, 2014.

15. Kawashima T, Kagawa S, Kobayashi N, Shirakiya Y, Umeoka T, Teraishi F, Taki M, Kyo S, Tanaka N and Fujiwara T: Telomerasespecific replication-selective virotherapy for human cancer. Clin Cancer Res 10: 285-292, 2004.

16. Kasuya H, Takeda S, Shimoyama S, Shikano T, Nomura N, Kanazumi N, Nomoto S, Sugimoto H and Nakao A: Oncolytic virus therapy - foreword. Curr Cancer Drug Targets 7: 123-125, 2007.

17. Zhang H, Wang H, Zhang J, Qian G, Niu B, Fan X, Lu J, Hoffman AR, Hu JF and Ge S: Enhanced therapeutic efficacy by simultaneously targeting two genetic defects in tumors. Mol Ther 17: 57-64, 2009.

18. Yan FC, Wang QQ, Ruan YH, Ma LJ, Jia JT and Jin KW: Establishment and biological characteristics of lung cancer cell line XWLC-05. Ai Zheng 26: 21-25, 2007 (In Chinese).

19. Anders M, Christian C, McMahon M, McCormick F and Korn WM: Inhibition of the Raf/MEK/ERK pathway up-regulates expression of the coxsackievirus and adenovirus receptor in cancer cells. Cancer Res 63: 2088-2095, 2003.

20. Song X, Zhou Y, Jia R, Xu X, Wang H, Hu J, Ge S and Fan X: Inhibition of retinoblastoma in vitro and in vivo with conditionally replicating oncolytic adenovirus H101. Invest Ophthalmol Vis Sci 51: 2626-2635, 2010.

21. Zhang C, Awasthi N, Schwarz MA, Hinz S and Schwarz RE: Superior antitumor activity of nanoparticle albumin-bound paclitaxel in experimental gastric cancer. PLoS One 8: e58037, 2013.

22. Liu TC and Kirn D: Gene therapy progress and prospects cancer: Oncolytic viruses. Gene Ther 15: 877-884, 2008.

23. Bischoff JR, Kirn DH, Williams A, Heise C, Horn S, Muna M, Ng L, Nye JA, Sampson-Johannes A, Fattaey A, et al: An adenovirus mutant that replicates selectively in p53-deficient human tumor cells. Science 274: 373-376, 1996.

24. Post DE, Khuri FR, Simons JW and Van Meir EG: Replicative oncolytic adenoviruses in multimodal cancer regimens. Hum Gene Ther 14: 933-946, 2003.
25. Ranki T, Särkioja M, Hakkarainen T, von Smitten K, Kanerva A and Hemminki A: Systemic efficacy of oncolytic adenoviruses in imagable orthotopic models of hormone refractory metastatic breast cancer. Int J Cancer 121: 165-174, 2007.

26. Rajecki M,Kanerva A,Stenman UH,Tenhunen M,KangasniemiL, Särkioja M, Ala-Opas MY, Alfthan H, Sankila A, Rintala E, et al: Treatment of prostate cancer with Ad5/3Delta24hCG allows non-invasive detection of the magnitude and persistence of virus replication in vivo. Mol Cancer Ther 6: 742-751, 2007.

27. Nemunaitis J, Tong AW, Nemunaitis M, Senzer N, Phadke AP, Bedell C, Adams N, Zhang YA, Maples PB, Chen S, et al: A phase I study of telomerase-specific replication competent oncolytic adenovirus (telomelysin) for various solid tumors. Mol Ther 18: 429-434, 2010

28. Yu W and Fang H: Clinical trials with oncolytic adenovirus in China. Curr Cancer Drug Targets 7: 141-148, 2007.

29. Freytag SO, Movsas B, Aref I, Stricker H, Peabody J, Pegg J, Zhang Y, Barton KN, Brown SL, Lu M, et al: Phase I trial of replication-competent adenovirus-mediated suicide gene therapy combined with IMRT for prostate cancer. Mol Ther 15: 1016-1023, 2007.

30. Yamamoto M, Davydova J, Wang M, Siegal GP, Krasnykh V, Vickers SM and Curiel DT: Infectivity enhanced, cyclooxygenase-2 promoter-based conditionally replicative adenovirus for pancreatic cancer. Gastroenterology 125: 1203-1218, 2003.

31. Ganly I, Kirn D, Eckhardt G, Rodriguez GI, Soutar DS, Otto R, Robertson AG, Park O, Gulley ML, Heise C, et al: A phase I study of Onyx-015, an E1B attenuated adenovirus, administered intratumorally to patients with recurrent head and neck cancer. Clin Cancer Res 6: 798-806, 2000.

32. Anders M, Rösch T, Küster K, Becker I, Höfler H, Stein HJ, Meining A, Wiedenmann B and Sarbia M: Expression and function of the coxsackie and adenovirus receptor in Barrett's esophagus and associated neoplasia. Cancer Gene Ther 16: 508-515, 2009

33. Brüning A, Stickeler E, Diederich D, Walz L, Rohleder H, Friese $\mathrm{K}$ and Runnebaum IB: Coxsackie and adenovirus receptor promotes adenocarcinoma cell survival and is expressionally activated after transition from preneoplastic precursor lesions to invasive adenocarcinomas. Clin Cancer Res 11: 43164320, 2005.

34. Qin M, Escuadro B, Dohadwala M, Sharma S and Batra RK: A novel role for the coxsackie adenovirus receptor in mediating tumor formation by lung cancer cells. Cancer Res 64: 6377-6380, 2004.

35. Fuxe J, Liu L, Malin S, Philipson L, Collins VP and Pettersson RF: Expression of the coxsackie and adenovirus receptor in human astrocytic tumors and xenografts. Int J Cancer 103: 723-729, 2003.

36. Yamamoto $\mathrm{M}$ and Curiel DT: Current issues and future directions of oncolytic adenoviruses. Mol Ther 18: 243-250, 2010.

37. Pesonen S, Kangasniemi L and Hemminki A: Oncolytic adenoviruses for the treatment of human cancer: Focus on translational and clinical data. Mol Pharm 8: 12-28, 2011. 